\title{
The Benefits of Correlative Scanning Electron - and Confocal Raman Microscopy for the Characterization of Polymers, 2D Materials and Lithium Oxides
}

\author{
Ute Schmidt $^{1 *}$, Wei Liu ${ }^{2}$, David Steinmetz ${ }^{1}$ and Stefanie Freitag ${ }^{3}$ \\ 1. WITec GmbH, Ulm, Germany. \\ 2. WITec Instruments Corp., Knoxwille, Tennessee, USA. \\ 3. Carl Zeiss Microscopy GmbH, Munich, Germany. \\ * Corresponding author: Ute.Schmidt@witec.de
}

The characterization of low-Z materials such as polymers, $2 \mathrm{D}$ materials, Li-oxides requires in most cases both, nondestructive high resolution imaging and chemical identification capabilities.

Scanning Electron Microscopy (SEM), based on the interaction of electrons with matter, provides highest resolution imaging capabilities on such materials without pre-treatment or coating when operated at acceleration voltages below $2 \mathrm{kV}$ [1]. The chemical composition of the materials, however, is difficult to analyze with energy or wavelength dispersive X-ray spectroscopy (EDS or WDS) due to the low acceleration voltage required for nondestructive imaging, the small sample volume of mono- or double-layer of 2D materials and the low atomic number $(Z<10)$ constituting the materials of interest [2]. Furthermore, properties of polymers are achieved mainly by changing the bonds between carbon and hydrogen atoms features which are undetectable by methods relaying on interactions of electrons with matter.

Raman spectroscopy, based on the interaction of photons with matter, is sensitive to the molecular and crystal structures of the analyzed materials. Its applications include chemical fingerprinting especially for polymers, structure and strain rates in films of 2D materials and oxidation states in lithium based oxides. By combining Raman spectroscopy with a confocal microscope, the distribution of various phases of materials can be imaged with diffraction limited optical resolution [3].

The integration of confocal Raman Imaging into the vacuum chamber of a SEM (RISE Microscopy) enables the complete characterization of materials with respect to high resolution imaging and chemical and structural composition of such materials $[3,4]$. The figures show examples of RISE measurements of three materials, revealing the de-mixing behavior in a PS-PMMA polymer blend (Fig. 1) [4], the structural properties of mono- and bi-layer graphene (Fig. 2) [5] and the composition of a $\mathrm{LiCo}_{\mathrm{x}} \mathrm{Ni}_{1-\mathrm{x}} \mathrm{O}_{2}$ particle after composite electroformation (Fig. 3) [6-8].

The aim of this contribution is to highlight the power of RISE Microscopy for the characterization of low-Z materials in terms of spatial resolution and chemical composition.

References:

[1] MD Frey in "Scanning Microscopy for Nanotechnology", ed. W Zhou, ZL Wang (Springer, New York) p. 101.

[2] DE Newbury and NWM Ritchie, Scanning 35 (2013), p. 141.

[3] "Confocal Raman Microscopy" ed. J Toporski, T Dieing, O Hollricher (Springer, Heidelberg).

[4] U Schmidt et al., Microsc. Microanal. 21 (2015), p. 2189.

[5] U Schmidt et al., Microsc. Microanal 23 (2017), p. 1748.

[6] T Sörgel et al., Journal of Power Sources 296 (2015) p. 70.

[7] RB Hadjean and JP Pereira-Ramos, Chem. Rev. 110 (2010), p. 1278.

[8] S Freitag et al., White paper in preparation. 

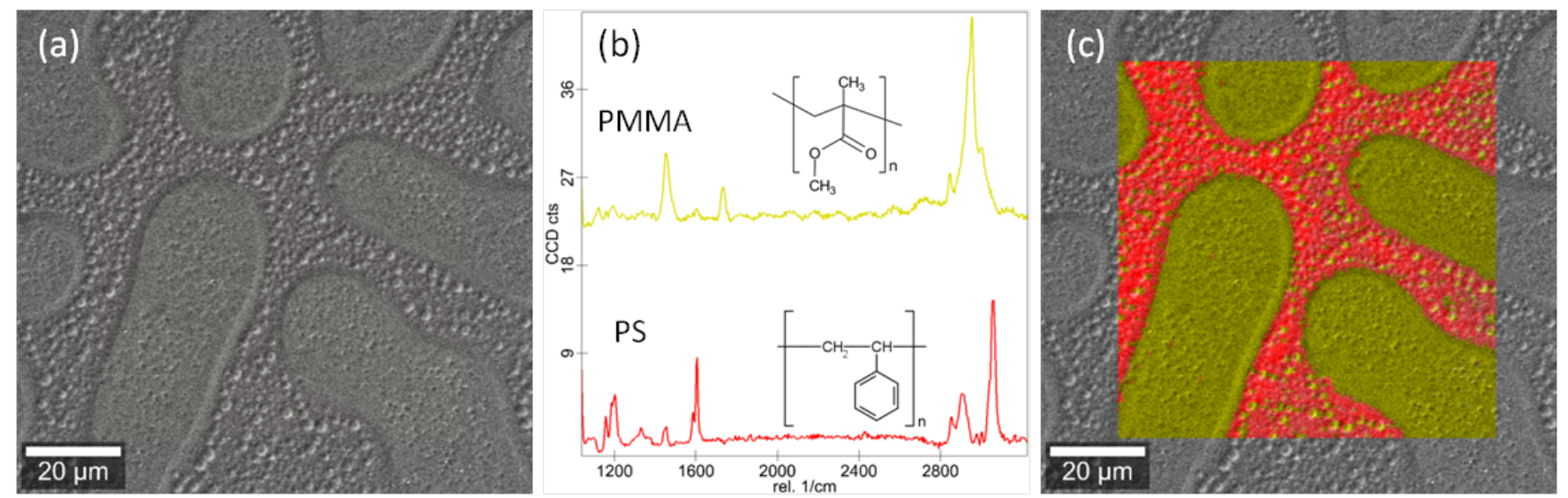

Figure 1. RISE imaging of a polymer blend of PS-PMMA: SEM image (a), color coded Raman spectra (b) and RISE image consisting of an overlay of Raman and SEM image revealing well separated domains of PS and PMMA (c).
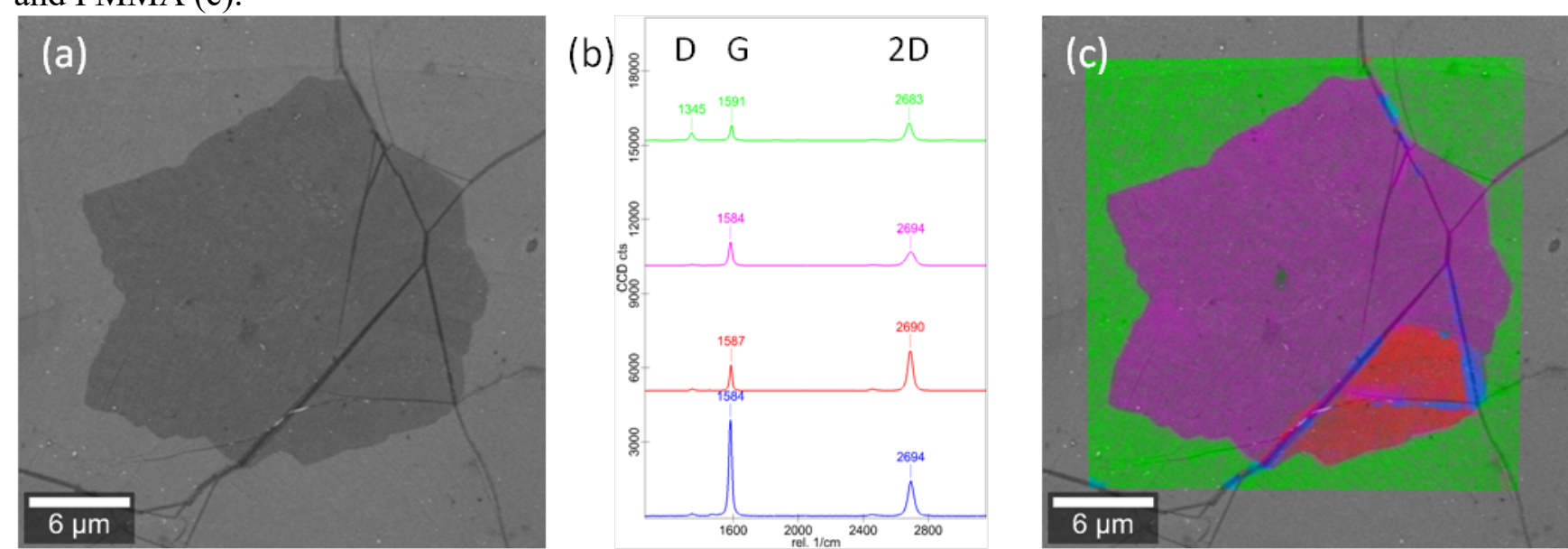

Figure 2. RISE imaging of mono- and double layer graphene: SEM image (a), color coded Raman spectra (b) and RISE image (c). From the position and intensity variations of the G and 2D band the number of graphene layers and their stacking can be deduced. The intensity of the D band reveals the defect density within the monolayer.
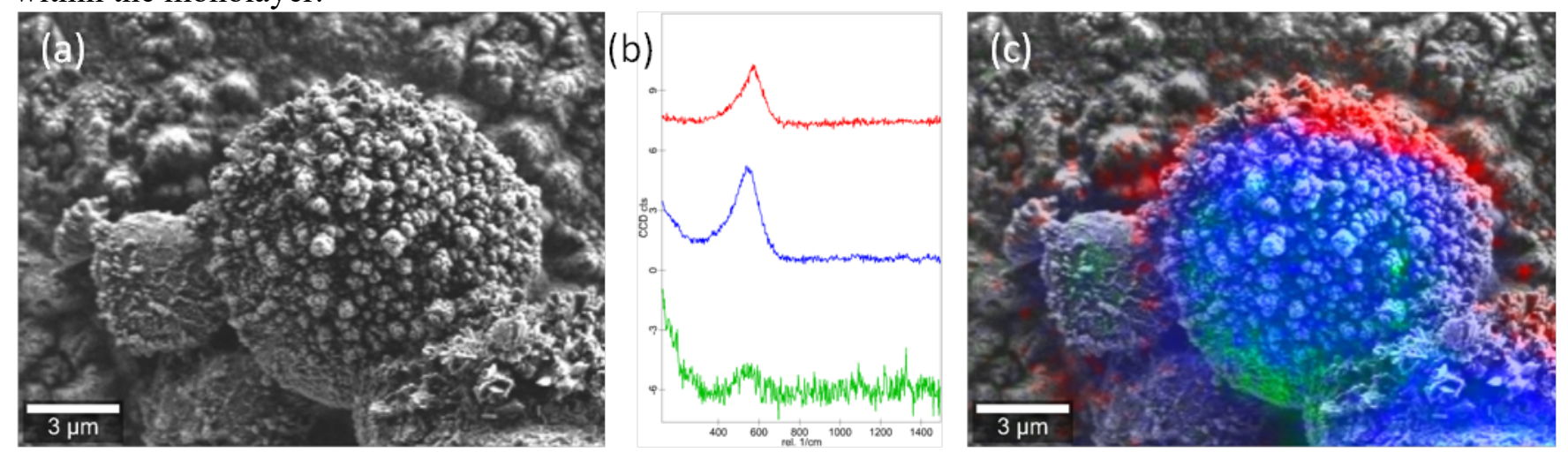

Fig. 3. RISE imaging of a $\mathrm{LiCoNiO}_{2}$ particle after composite electroformation [6]: SEM image (a), color coded Raman spectra (b) and RISE image (c). The particles consist of a core-shell structure with varying content of $\mathrm{Ni}$ and $\mathrm{Co}$ : red - high $\mathrm{Co}$, blue - intermediate Co, green - no Co content [7]. 